\title{
Divergent Integrals Treatment in Quantum Field Theory
}

\author{
S. R. Gobira*1@ and M. D. B. de Melo" \\ ${ }^{1}$ Universidade Federal do Tocantins, Palmas, TO, Brasil.
}

Received on September 11, 2020. Revised on November 06, 2020. Accepted on November 17, 2020.

\begin{abstract}
This paper presents a brief review on regularization methods and shows that the combination of two techniques could preserve symmetries in all orders of the Perturbation Theory. We will see, with two simple examples in the one-loop-order, what a regularization method needs to preserve symmetries. Furthermore, it will be shown that the problems with symmetry violations by regularization methods are related to the consequences of not being able to simply shift a variable in these divergent integrals. To facilitate the analysis between methods and provide analytical expressions for the finite parts of amplitudes, it was systematized in terms of a set of special functions. In the Appendix we introduce these functions and then cases of specific interest, functions continuity and some useful limits will be presented.
\end{abstract}

Keywords: Propagator, Regularization.

\section{Introduction}

In Quantum Field Theory (QFT), problems with divergent integrals are common in several models that propose to describe interacting physical particles. The procedure that allows the elimination of these infinities in favor of a redefinition of the parameters of a Lagrangian is known as Renormalization Theory. It is crucial for the applicability of Quantum Field Theories in general. The process of renormalizing a theory begins with the study of the renormalizability of that theory by using dimensional arguments and power counting to study the basic divergence properties of the model. The Bogoliubov-Parasiuk-Hepp-Zimmermann (BPHZ) theorem [1 3] states that all divergences from quantum field theories can be removed by building counter-terms for the superficially divergent Feynman graphs. Next, we have to choose a regularization method to deal with divergent integrals. Historically, during the construction of Quantum Electrodynamics (QED) [4, 5], Dimensional Regularization (DR) [6, 7] was what made it possible to renormalize QED while preserving, in each order, the Lagrangian symmetries. It was undoubtedly an advance in the battle against the infinite, which also infest other QFT. Despite the enormous success of DR, it was soon realized that this method presents difficulties in some contexts, for example, when objects such as the $\gamma^{5}$ matrix or anti symmetric tensors $\epsilon_{\mu \nu \alpha}$ are present in the theory. Appropriate extensions of DR must be constructed so that the properties of these objects do not clash with the idea of analytical continuation in the space-time dimension. During the text that follows we consider only the cases where the Perturbation Theory and, consequently, Feynman's rules apply. We show, with

\footnotetext{
* Correspondence email address: gobira@uft.edu.br
}

a simple example, that only three relations between divergent integrals are needed to preserve symmetries in the first order in a renormalizable Lagrangian, such as that of QED, in four dimensions. In addition, it will be clear that the problems with violations of symmetries by regularization methods are related to the consequences of not being able to do "shifts" in the integration variable in divergent integrals. For this we will consider three methods of regularization. The first fails to preserve symmetries, the second respects symmetries but presents difficulties with the spacetime dimensionality and a third that, if we take some care, respects symmetries and can be used without restriction in any space-time dimension. The methods are: the regularization of Pauli Villars [8], a pioneer of regularization methods; Dimensional Regularization (DR) that preserves the symmetries of QED; and the Implicit Regularization Method (IR) 9-12]. In the latter we say that the integral is regularized for the purposes of algebraic manipulations, but we do not need to use any regularization method explicitly. The results are presented in terms of a sum of terms proportional to the implicitly regularized integrals, which do not depend on the external moment, and a finite part that is directly integrated. This procedure will be made clear in the sections below where the result of the explicit calculation of some amplitudes are shown. The terms containing the divergent integrals can be identified as being the counterterms necessary to renormalize the fields, masses and coupling constants of a theory in a certain order of the Perturbation Theory. On the other hand, using a result in terms of regularized integrals [9], one can reproduce any result of other regularization methods, showing where a given method fails or succeeds in preserving the symmetries of a theory. This will be clear from the procedures in the sections below where we will show in 
detail the identities that some divergent integrals must obey in order to preserve the symmetries contained in a Lagrangian, in the "one loop" approximation and in a space with four dimensions.

In this paper we use the standard notation found in the QFT literature 13 and it is organized as follows: in the next section we will give an example of a divergent amplitude where the result of the calculation will be presented in terms of the $\mathrm{Z}$ and $\mathrm{Y}$ functions. In this example, the relationship between shifting integrals and possible ambiguities in the calculation of two-point Green functions will be shown. Three different regularization methods will be used so that we can clearly analyze their differences. In the penultimate section we will show that the possible ambiguities generated by not being able to shift divergent integrals are related to violations of symmetries contained in the original Lagrangian. Finally, we will have the conclusions and bibliographic references. In the Appendix we will define the functions which we call $\mathrm{Z}$ and $\mathrm{Y}$ and explicitly present some important results. It will also contain some limits, useful for analyzing the calculations resulting from different regularization methods.

\section{Comparing Regularization Methods}

In renormalizable QFTs which gauge symmetry, like QED, we have the following procedure: Lagrangian with original symmetries $\rightarrow$ divergent corrections $\rightarrow$ regularization methods $\rightarrow$ renormalized Lagrangian. In this procedure, we need to be careful so that the renormalized Lagrangian continues with its original symmetries. We will now show where the terms responsible for symmetries violations in divergent amplitudes come from. Let us use a simple example of divergent amplitude to show how the regularization methods work, focusing on the Pauli-Villars Regularization, Dimensional Regularization and Implicit Regularization Methods.

We will use an arbitrary parameter " $\alpha$ " for the internal momentum of the loop to highlight a possible amplitude dependency with the choice of momentum routing. The parameter " $\alpha$ " is a real number and, by choosing a value for " $\alpha$ ", we select one possible label for each internal line in the diagram. Let us consider the diagram in Figure 1. We can see that, in principle, " $\alpha$ " can have any value since none violates the momentum energy conservation at the vertices. The external lines represent a scalar field,

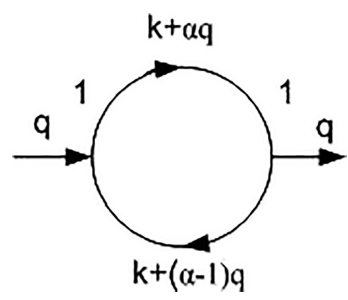

Figure 1: Fermion loop. so we will use the notation $T_{s s}$ indicating that we have two external scalar fields. The internal lines correspond to spin $\frac{1}{2}$ and mass $m$ fermions. We will use the notation $\not p=\gamma^{\mu} p_{\mu}$. The Feynman's rules for this diagram give us the amplitude:

$$
\begin{aligned}
T_{s s}= & \int \frac{d^{4} k}{(2 \pi)^{4}} T r \\
& \times\left[\frac{1}{[(\not k+\alpha \not q)-m]} \frac{1}{[\not k+(\alpha-1) \not 1-m]}\right]
\end{aligned}
$$

Note that the integral is quadratic divergent. To see this, just count the power of the integration variable in the numerator and denominator, as the integral's degree of divergence $\mathrm{D}$ will be the power of the numerator subtracting the power of the denominator. If it were finite, or logarithmically divergent, we could change the integration variable to eliminate the alpha parameter. By means of the redefinition

$$
\not k^{\prime}=\not k+\alpha \not 1
$$

the parameter " $\alpha$ " in (1) will be eliminated. We will now present the results for this amplitude using three methods in chronological order in the evolution of regularization methods.

\subsection{Pauli-Villars Regularization}

The covariant regularization or Pauli-Villars regularization consists of modifying the integrals by transforming the propagator as follows

$$
\frac{1}{k^{2}-m^{2}} \rightarrow \frac{1}{k^{2}-m^{2}}+\sum_{i} \frac{a_{i}}{k^{2}-\Lambda_{i}^{2}}
$$

where $\Lambda$ is the regularization parameter that must satisfy the condition $\Lambda_{i}^{2} \gg m^{2}$. The effect of this modification is, in short, equivalent to multiplying the integral by functions of the type $\left(\frac{m^{2}-\Lambda_{i}^{2}}{k^{2}-\Lambda_{i}^{2}}\right)$. That way, when we take the limit $\Lambda_{i}^{2} \rightarrow \infty$ these functions tend to one. Multiply as many functions as necessary so that the integral becomes finite. We generally use the same regularization parameter for all functions, that is, we multiply the integrand by $\left(\frac{m^{2}-\Lambda^{2}}{k^{2}-\Lambda^{2}}\right)^{n}$ where $n+1$ is the number of times the operation is necessary.

So let us apply this to our example. After taking the trace of the Dirac matrices, regularizing the integrals and integrating directly we obtain:

$$
\begin{aligned}
T_{s s}= & \frac{2 i}{(4 \pi)^{2}}\left\{\left(-m^{2}\right)^{-1}\left(m^{2}-\Lambda^{2}\right)^{2}\right. \\
& \cdot\left[Y_{0}\left(\Lambda^{2}, m^{2}, \alpha^{2} q^{2}\right)+Y_{0}\left(\Lambda^{2}, m^{2},(\alpha-1)^{2} q^{2}\right)\right] \\
& \left.+\left[4 m^{2}-q^{2}\right]\left[Z_{0}\left(\Lambda^{2}, m^{2}, q^{2}\right)-Z_{0}\left(m^{2}, q^{2}\right)\right]\right\}
\end{aligned}
$$

Note the explicit alpha dependency on the second line of $(3)$. 


\subsection{Dimensional Regularization}

This method consists of modifying the dimensionality of the integrals, which are originally in a four-dimensional space to a $2 \omega$ dimensional space, so that they become finite. We solve the integral in that space and then return to the original space taking the limit of $\omega$ tending to two. When this limit is taken we get the sum of finite terms and divergent terms as in the previous method. The gamma matrices are defined in a $2 \omega$ dimensional space. The metric tensor, in this space, is defined in such a way that $g^{\mu \nu} g_{\mu \nu}=2 \omega$ and so we have a set of gamma matrices that satisfy the anticommutation relation $\left\{\gamma^{\mu}, \gamma^{\nu}\right\}=2 g^{\mu \nu}$. The gamma matrices and the matrix unit 1 are $f(2 \omega) \times f(2 \omega)$ matrices. The trace of a gamma matrix or a product of an odd number of these matrices is always equal to zero. Taking the trace in this space and following a path analogous to that made in four-dimensional space we obtain the final expression:

$$
\begin{aligned}
T_{s s}= & \frac{i f(2 \omega)}{2(4 \pi)^{\omega}}\left\{2\left(-m^{2}\right)^{\omega-1}\left[\frac{1}{(2-\omega)(1-\omega)}+\frac{A}{(1-\omega)}\right]\right. \\
& \left.+\left[4 m^{2}-q^{2}\right]\left[\frac{1}{2-\omega}-Z_{0}\left(m^{2}, q^{2}\right)+\ln \left(m^{2}\right)+A\right]\right\}
\end{aligned}
$$

where "A" is a constant that appears with the Gamma Function expansion. We only consider terms up to the first order in the constant "A" in the expansion. Note that the result is automatically independent of " $\alpha$ " as it should be. This was already expected because, in an omega dimensional space, the integrals are finite and therefore we can do (2) before taking the trace in (1) and eliminate the dependency with parameter " $\alpha$ ".

\subsection{Implicit Regularization Method}

The Implicit Regularization Method is based on algebraic identities to rewrite the amplitude as a sum of integrals and follows the steps below:

- After taking the trace of the Dirac matrices and identifying the divergent integrals, each of these integrals is manipulated only at the level of the integral function, until all dependence on the external momenta is strictly contained in finite integrals. The identity below, as we can see, can be used successively in order to isolate the internal momenta from the external ones.

$$
\begin{aligned}
& \frac{1}{\left[\left(p-k_{j}\right)^{2}-m^{2}\right]} \\
& \quad=\frac{1}{k_{j}^{2}-m^{2}}+\frac{2 p \cdot k_{j}-p^{2}}{\left(k_{j}^{2}-m^{2}\right)}\left\{\frac{1}{\left[\left(p-k_{j}\right)^{2}-m^{2}\right]}\right\}
\end{aligned}
$$

- Finite integrals must be integrated without restrictions.
- In each order of the Perturbation Theory, it is necessary that some differences between divergent integrals, totally independent of the external momenta, vanish. This procedure ensures the invariance of the integral result with the variable changing 2. In one loop order, as we will see, the divergent integrals, independent of the external momenta, are reduced to basic integrals $I_{\text {quad }}$ and $I_{\log }$ :

$$
\begin{aligned}
I_{\text {quad }}\left(m^{2}\right) & =\int \frac{d^{4} k}{(2 \pi)^{4}} \frac{1}{\left[k^{2}-m^{2}\right]} \\
I_{\log }\left(m^{2}\right) & =\int \frac{d^{4} k}{(2 \pi)^{4}} \frac{1}{\left[k^{2}-m^{2}\right]^{2}}
\end{aligned}
$$

The application of the method provides the following result for equation (1)

$$
\begin{aligned}
T_{\text {ss }}= & 4\left[I_{\text {quad }}\left(m^{2}\right)+\frac{\left[4 m^{2}-q^{2}\right]}{2} I_{\log }\left(m^{2}\right)\right. \\
& \left.-\frac{\left[4 m^{2}-q^{2}\right]}{2} \frac{i}{(4 \pi)^{2}} Z_{0}\left(m^{2}, q^{2}\right)\right] \\
& -2\left(2 \alpha^{2}-2 \alpha-1\right) \\
& \left\{q^{2} I_{\log }\left(m^{2}\right)-4 q^{\mu} q^{\nu} \int \frac{d^{4} k}{(2 \pi)^{4}} \frac{k^{\mu} k^{\nu}}{\left[k^{2}-m^{2}\right]^{3}}\right\} .
\end{aligned}
$$

We can see that the only term that depends on the " $\alpha$ " parameter is the last one. The amplitude does not depend on " $\alpha$ " parameter if the difference between the divergent integrals in the last term in (8) is zero, that is

$$
\frac{1}{4} g^{\mu \nu} I_{\log }\left(m^{2}\right)=\int \frac{d^{4} k}{(2 \pi)^{4}} \frac{k^{\mu} k^{\nu}}{\left[k^{2}-m^{2}\right]^{3}}
$$

Any regularization method that succeeds satisfying equality (9) will be a regularization method that preserves symmetries, as will be clear in the next section. The DR satisfies equality 9 as we can easily verify.

We can explicitly see that by Pauli Villars regularization equality 9 is not achieved, since

$$
\begin{aligned}
& \int \frac{d^{4} k}{(2 \pi)^{4}} \frac{\left(m^{2}-\Lambda^{2}\right)}{\left[k^{2}-m^{2}\right]^{2}\left[k^{2}-\Lambda^{2}\right]} \\
& \quad=\frac{i}{(4 \pi)^{2}} \cdot \frac{\left(\Lambda^{2}-m^{2}\right)}{\Lambda^{2}} Y_{0}\left(m^{2}, \Lambda^{2}, 0\right)
\end{aligned}
$$

and

$$
\begin{aligned}
\int \frac{d^{4} k}{(2 \pi)^{4}} \frac{k^{\mu} k^{\nu}\left(m^{2}-\Lambda^{2}\right)}{\left[k^{2}-m^{2}\right]^{3}\left[k^{2}-\Lambda^{2}\right]} \\
=\frac{i}{(4 \pi)^{2}} \\
\quad \cdot \frac{\left(\Lambda^{2}-m^{2}\right)}{4 \Lambda^{2}} g_{\mu \nu}\left[Y_{0}\left(m^{2}, \Lambda^{2}, 0\right)-Y_{1}\left(m^{2}, \Lambda^{2}, 0\right)\right]
\end{aligned}
$$




\section{Symmetry and Divergent Integrals}

Now let us consider a renormalizable theory, in spacetime dimension $\mathrm{n}+1$ that can be expanded into a Perturbative series, such as QED. In each order of the Perturbation Theory, how can we find the surface terms, that is, the differences between divergent integrals in order to, with the choice of momentum routing, ensure the invariance of the integrals result? For the renormalization of a given theory, we must identify these differences between integrals, with the same degree of divergence in each order. We can see that the use of identity (5) will gradually lower the degree of divergence of the integral. Therefore, if we study a process with the highest degree of divergence of the theory, all differences between integrals of the lowest orders will appear.

Now we consider the QED Lagrangian. In the case of QED [11, we chose the diagram in the Figure 2 because it has a cubic divergence. Another reason for choosing this diagram is that it is related to the following theorem and the following symmetry relation. In the Furry theorem [14, the vacuum expectation value of any odd number of electromagnetic currents must vanish. As a consequence, the fermionic amplitude of a point coupled with an odd number of external photons should cancel itself out because it possesses an odd number of vector indexes. The Furry theorem is a consequence of the charge conjugation symmetry in QED. This ensures that there are no physical processes that evolve only photons and have an odd number of external photons. In the case of one external photon, we have

$$
T_{\mu}=0
$$

On the other hand, the conservation of the vector current (gauge invariance), that is $\partial^{\mu} J_{\mu}(x)=0$, or in the momentum space $q^{\mu} J_{\mu}(q)=0$, will lead to a set of QED Ward identities. For our specific case, the Ward identity is

$$
q^{\mu} T_{\mu}=0
$$

The conservation of the vector current, by the Noether

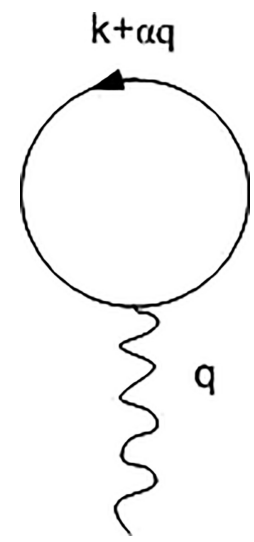

Figure 2: Tadpole diagram. theorem, guarantees the conservation of the electric charge.

Now let us consider the diagram in Figure 2 The QED Feynman rules result in

$$
T_{\mu}^{V}=e \int_{\Lambda} \frac{d^{4} k}{(2 \pi)^{4}} \operatorname{Tr}\left\{\gamma_{\mu} \frac{1}{[(\not k+\alpha \not q)-m]}\right\}
$$

Again, if we do $k^{\prime}=\not k+\alpha \not q$ the alfa dependence disappears.

The integral (14) has a cubic divergence as we can see. After taking the trace of the Dirac matrices, using the identity (5) and directly integrating the finite parts, the amplitude can be expressed as:

$$
\begin{aligned}
T_{V}^{\mu}= & 4 e\left\{\alpha q_{\mu} I_{\text {quad }}\left(m^{2}\right)-\alpha q_{\nu} \int_{\Lambda} \frac{d^{4} k}{(2 \pi)^{4}} \frac{2 k^{\mu} k^{\nu}}{\left(k^{2}-m^{2}\right)^{2}}\right. \\
& +\alpha^{3} q^{2} q_{\nu}\left[\int_{\Lambda} \frac{d^{4} k}{(2 \pi)^{4}} \frac{4 k^{\mu} k^{\nu}}{\left(k^{2}-m^{2}\right)^{3}}-g_{\mu \nu} I_{\log }\left(m^{2}\right)\right] \\
& +4 \alpha^{3} q_{\nu} q_{\rho} q_{\sigma} \\
& {\left.\left[\int_{\Lambda} \frac{d^{4} k}{(2 \pi)^{4}} \frac{g^{\rho \sigma} k^{\mu} k^{\nu}}{\left(k^{2}-m^{2}\right)^{3}}-\int_{\Lambda} \frac{d^{4} k}{(2 \pi)^{4}} \frac{2 k^{\mu} k^{\nu} k^{\rho} k^{\sigma}}{\left(k^{2}-m^{2}\right)^{4}}\right]\right\} }
\end{aligned}
$$

The conditions

$$
\begin{gathered}
g_{\mu \nu} I_{\text {quad }}\left(m^{2}\right)=\int_{\Lambda} \frac{d^{4} k}{(2 \pi)^{4}} \frac{2 k_{\mu} k_{\nu}}{\left(k^{2}-m^{2}\right)^{2}} \\
\int_{\Lambda} \frac{d^{4} k}{(2 \pi)^{4}} \frac{4 k_{\mu} k_{\nu}}{\left(k^{2}-m^{2}\right)^{3}}=g_{\mu \nu} I_{\log }\left(m^{2}\right) \\
\int_{\Lambda} \frac{d^{4} k}{(2 \pi)^{4}} \frac{g^{\rho \alpha} k_{\mu} k_{\nu}}{\left(k^{2}-m^{2}\right)^{3}}=\int_{\Lambda} \frac{d^{4} k}{(2 \pi)^{4}} \frac{2 k_{\mu} k_{\nu} k_{\rho} k_{\sigma}}{\left(k^{2}-m^{2}\right)^{4}}
\end{gathered}
$$

are necessary for the amplitude to be alpha independent and equally null. Notice that, by Dimensional Regularization, the amplitude is also zero. Through Dimensional Regularization (16), (17) and (18) are also satisfied. Therefore, as became clear in this example, one of the reasons behind the consistency of the Dimensional Regularization is this.

The Pauli-Villars Regularization will not satisfy them as we can see below: The equality $(9)$ is the same as that found in (17), and we show in 10 and 111 that the integrals regularized by Pauli-Villars will not satisfy (9). We need to check only relations $(16)$ and $(18)$. The left side of (18) regularized results in (11). On the right side of (18), we have

$$
\begin{aligned}
\int \frac{d^{4} k}{(2 \pi)^{4}} \frac{k_{\mu} k_{\nu} k_{\alpha} k_{\beta}\left(m^{2}-\Lambda^{2}\right)}{\left[k^{2}-m^{2}\right]^{4}\left[k^{2}-\Lambda^{2}\right]} \\
=\frac{i}{(4 \pi)^{2}} g_{\mu \nu \alpha \beta} \frac{\left(m^{2}-\Lambda^{2}\right)}{-4 \Lambda^{2}} \\
\cdot\left[Y_{0}\left(m^{2}, \Lambda^{2}, 0\right)-2 Y_{1}\left(m^{2}, \Lambda^{2}, 0\right)+Y_{2}\left(m^{2}, \Lambda^{2}, 0\right)\right]
\end{aligned}
$$


where $g_{\mu \nu \rho \sigma}=g_{\mu \nu} g_{\rho \sigma}+g_{\rho \mu} g_{\nu \sigma}+g_{\rho \nu} g_{\mu \sigma}$. Finally, on the right side of (16), we have

$$
\begin{aligned}
& \int \frac{d^{4} k}{(2 \pi)^{4}} \frac{k_{\mu} k_{\nu}\left(m^{2}-\Lambda^{2}\right)^{2}}{\left[k^{2}-m^{2}\right]^{2}\left[k^{2}-\Lambda^{2}\right]^{2}} \\
& \quad=\frac{i}{(4 \pi)^{2}} \cdot \frac{\left(m^{2}-\Lambda^{2}\right)^{2}}{-m^{2}} g_{\mu \nu} Y_{1}\left(m^{2}, \Lambda^{2}, 0\right)
\end{aligned}
$$

and on the left side of 16

$$
\begin{aligned}
& \int \frac{d^{4} k}{(2 \pi)^{4}} \frac{\left(m^{2}-\Lambda^{2}\right)^{2}}{\left[k^{2}-m^{2}\right]\left[k^{2}-\Lambda^{2}\right]^{2}} \\
& \quad=\frac{i}{(4 \pi)^{2}} \cdot \frac{\left(m^{2}-\Lambda^{2}\right)^{2}}{-m^{2}} Y_{0}\left(m^{2}, \Lambda^{2}, 0\right)
\end{aligned}
$$

As we can see, the Pauli-Villars regularization violates the three conditions necessary to guarantee the symmetries and the momentum label invariance.

\section{Conclusions}

We show, in a QED example, that the same three relations, equations (16), (17) and (18), that guarantee the invariance under momentum routing, also ensure the validity of the Furry theorem (charge conjugation symmetry) and the conservation of the electric charge (gauge symmetry). The origin of the symmetry violations introduced by regularization methods can be mapped through relations between divergent integrals. These relations appear as differences between integrals with the same degree of divergence and must be canceled so that the amplitude is independent of the choice of the loop moment labeling. The same differences between divergent integrals are responsible for symmetry violations contained in the original Lagrangian. Note that equality $(9)$ is the same as that found in (17). In the first order of approximation, in four dimensions, only three conditions must be satisfied so that the amplitude is alpha independent and consequently preserves all symmetries. The Implicit Regularization Method will only be consistent in all orders if we are able to cancel all these surface terms in each order. The use of identity (5) will gradually lower the integral's degree of divergence. Therefore, if we study a process of the theory with the highest degree of divergence, all differences between integrals of the lowest orders will appear. In all orders of Perturbation Theory we can see that a combination of the Implicit Regularization Method with any regularization method that cancels out unwanted surface terms, will always be able to preserve the original symmetries of a Lagrangian.

\section{A. The Functions $Z_{k}$ and $Y_{k}$}

The integrals, divergent or finite, that we obtain in the calculation of Green's functions in QFT, in the first order of the perturbative series, when treated by any regularization method or directly integrated, lead to results that can be expressed in terms of a particular class of functions. In this appendix we will define these functions and present some of their relevant limits. This procedure facilitates the analysis of the results presented in this work. An important physical aspect that can be read in these results is the mandatory existence of an imaginary part in the region where the square module of the four-vector moment energy is greater than the sum of the squares of the masses. Other important physical aspects are the behavior of these functions in the mass shell and their continuity in all regions of momentum space.

Let functions $Z_{k}\left(\lambda_{1}^{2}, \lambda_{2}^{2}, p^{2}\right)$ and $Y_{k}\left(\lambda_{1}^{2}, \lambda_{2}^{2}, p^{2}\right)$ of the constants $\lambda_{1}^{2}$ and $\lambda_{2}^{2}$ represent two different masses and the module square of the moment-energy four-vector $p^{2}$. Let us first define them in a general way and then study them in the particular cases where the results of amplitudes calculated in QFT can be expressed, as shown in 9] and [12.

The $Z_{k}$ and $Y_{k}$ functions are defined as:

$$
\begin{aligned}
& Z_{k}\left(\lambda_{1}^{2}, \lambda_{2}^{2}, p^{2}\right) \\
& \quad=\int_{0}^{1} d z \ln \left[\frac{p^{2} z(1-z)+z\left(\lambda_{1}^{2}-\lambda_{2}^{2}\right)-\lambda_{1}^{2}}{\left(-\lambda_{2}^{2}\right)}\right] z^{k} \\
& Y_{k}\left(\lambda_{1}^{2}, \lambda_{2}^{2}, p^{2}\right) \\
& \quad=\int_{0}^{1} d z \frac{z^{k}(1-z)\left(-\lambda_{2}^{2}\right)}{\left[p^{2} z(1-z)+z\left(\lambda_{1}^{2}-\lambda_{2}^{2}\right)-\lambda_{1}^{2}\right]}
\end{aligned}
$$

$k=0, \pm 1, \pm 2 \ldots$ These functions, as we can see from the expression above, are related by:

$$
Y_{k+1}\left(\lambda_{1}^{2}, \lambda_{2}^{2}, p^{2}\right)=-\lambda_{2}^{2} \frac{\partial}{\partial p^{2}} Z_{k}\left(\lambda_{1}^{2}, \lambda_{2}^{2}, p^{2}\right)
$$

When we calculate these integrals, for $k=0,1,2$ we get as results:

$$
\begin{aligned}
Z_{0}= & -\frac{1}{2}\left[\frac{\left(p^{2}-\lambda_{2}^{2}+\lambda_{1}^{2}\right)}{p^{2}} \ln \left(\frac{\lambda_{2}^{2}}{\lambda_{1}^{2}}\right)+\frac{F \cdot G}{p^{2}}+4\right] \\
Z_{1}= & -\frac{1}{2}\left\{\left[\frac{\left(p^{2}-\lambda_{2}^{2}+\lambda_{1}^{2}\right)^{2}}{2 p^{4}}-\frac{\lambda_{1}^{2}}{p^{2}}\right] \ln \left(\frac{\lambda_{2}^{2}}{\lambda_{1}^{2}}\right)\right. \\
& \left.+\frac{\left(p^{2}-\lambda_{2}^{2}+\lambda_{1}^{2}\right)}{p^{2}}+1+\frac{\left(p^{2}-\lambda_{2}^{2}+\lambda_{1}^{2}\right)}{2 p^{4}} F \cdot G\right\} \\
Z_{2}= & -\frac{1}{3}\left\{\frac{1}{2}\left[\frac{\left(p^{2}-\lambda_{2}^{2}+\lambda_{1}^{2}\right)^{3}}{p^{6}}-3 \lambda_{1}^{2} \frac{\left(p^{2}-\lambda_{2}^{2}+\lambda_{1}^{2}\right)}{p^{4}}\right]\right. \\
& \ln \left(\frac{\lambda_{2}^{2}}{\lambda_{1}^{2}}\right) \\
& +\frac{\left(p^{2}-\lambda_{2}^{2}+\lambda_{1}^{2}\right)^{2}}{p^{4}}+\frac{\left(p^{2}-\lambda_{2}^{2}+\lambda_{1}^{2}\right)}{2 p^{2}}-2 \frac{\lambda_{1}^{2}}{p^{2}}+\frac{2}{3}
\end{aligned}
$$




$$
\begin{aligned}
& \left.+\frac{1}{2}\left[\frac{\left(p^{2}-\lambda_{2}^{2}+\lambda_{1}^{2}\right)^{2}}{p^{6}}-\frac{\lambda_{1}^{2}}{p^{4}}\right] F \cdot G\right\} \\
Y_{0}= & \frac{\lambda_{2}^{2}}{2 p^{2}}\left\{\ln \left(\frac{\lambda_{2}^{2}}{\lambda_{1}^{2}}\right)+\left(p^{2}-\lambda_{2}^{2}+\lambda_{1}^{2}\right) \frac{G}{F}\right\} \\
Y_{1}= & \frac{\lambda_{2}^{2}}{2 p^{2}}\left\{\left[1-\frac{\left(p^{2}-\lambda_{2}^{2}+\lambda_{1}^{2}\right)}{p^{2}}\right] \ln \left(\frac{\lambda_{2}^{2}}{\lambda_{1}^{2}}\right)\right. \\
& +\left[\left(p^{2}-\lambda_{2}^{2}+\lambda_{1}^{2}\right)+2 \lambda_{1}^{2}\right. \\
& \left.\left.-\frac{\left(p^{2}-\lambda_{2}^{2}+\lambda_{1}^{2}\right)^{2}}{p^{2}}-2\right] \frac{G}{F}\right\} \\
Y_{2}= & \frac{\lambda_{2}^{2}}{2 p^{2}}\left\{\frac { 1 } { 2 } \left[\frac{\left(p^{2}-\lambda_{2}^{2}+\lambda_{1}^{2}\right)^{2}}{p^{4}}\right.\right. \\
& \left.+\frac{\left(p^{2}-\lambda_{2}^{2}+\lambda_{1}^{2}\right)}{p^{2}}-\frac{\lambda_{1}^{2}}{p^{2}}\right] \\
& \ln \left(\frac{\lambda_{2}^{2}}{\lambda_{1}^{2}}\right)+1+\frac{2\left(p^{2}-\lambda_{2}^{2}+\lambda_{1}^{2}\right)}{p^{2}} \\
& +\left[\frac{\left(p^{2}-\lambda_{2}^{2}+\lambda_{1}^{2}\right)^{3}}{p^{4}}+\frac{\left(p^{2}-\lambda_{2}^{2}+\lambda_{1}^{2}\right)^{2}}{p^{2}}\right. \\
& \left.\left.-3 \lambda_{1}^{2} \frac{\left(p^{2}-\lambda_{2}^{2}+\lambda_{1}^{2}\right)}{p^{2}}-2 \frac{\lambda_{1}^{2}}{p^{2}}\right] \frac{G}{F}\right\}
\end{aligned}
$$

where $F=F\left(\lambda_{1}, \lambda_{2}, p^{2}\right)$ and $G=G\left(\lambda_{1}, \lambda_{2}, p^{2}\right)$. They are functions defined in three distinct regions:

region 1: $p^{2}<\left(\lambda_{1}-\lambda_{2}\right)^{2}$,

$$
\begin{aligned}
& F=-\sqrt{\left(\lambda_{1}-\lambda_{2}\right)^{2}-p^{2}} \sqrt{\left(\lambda_{1}+\lambda_{2}\right)^{2}-p^{2}} \\
& G=2 \ln \left[\frac{\sqrt{\left(\lambda_{1}+\lambda_{2}\right)^{2}-p^{2}}-\sqrt{\left(\lambda_{1}-\lambda_{2}\right)^{2}-p^{2}}}{\sqrt{\left(\lambda_{1}+\lambda_{2}\right)^{2}-p^{2}}+\sqrt{\left(\lambda_{1}-\lambda_{2}\right)^{2}-p^{2}}}\right]
\end{aligned}
$$

region 2: $p^{2}>\left(\lambda_{1}+\lambda_{2}\right)^{2}$,

$$
\begin{aligned}
& F=\sqrt{p^{2}-\left(\lambda_{1}-\lambda_{2}\right)^{2}} \sqrt{p^{2}-\left(\lambda_{1}+\lambda_{2}\right)^{2}} \\
& G=2 \ln \left[\frac{\sqrt{p^{2}-\left(\lambda_{1}-\lambda_{2}\right)^{2}}-\sqrt{p^{2}-\left(\lambda_{1}+\lambda_{2}\right)^{2}}}{\sqrt{p^{2}-\left(\lambda_{1}-\lambda_{2}\right)^{2}}+\sqrt{p^{2}-\left(\lambda_{1}+\lambda_{2}\right)^{2}}}\right]+2 i \pi
\end{aligned}
$$

region 3: $\left(\lambda_{1}-\lambda_{2}\right)^{2}<p^{2}<\left(\lambda_{1}+\lambda_{2}\right)^{2}$,

$$
\begin{aligned}
& F=i \sqrt{p^{2}-\left(\lambda_{1}-\lambda_{2}\right)^{2}} \sqrt{\left(\lambda_{1}+\lambda_{2}\right)^{2}-p^{2}} \\
& G=-4 i \arctan \left[\frac{\sqrt{\left(\lambda_{1}+\lambda_{2}\right)^{2}-p^{2}}}{\sqrt{p^{2}-\left(\lambda_{1}-\lambda_{2}\right)^{2}}}\right]
\end{aligned}
$$

The study of the behavior of these functions at points $p^{2}=\left(\lambda_{1}-\lambda_{2}\right)^{2}$ and $p^{2}=\left(\lambda_{1}+\lambda_{2}\right)^{2}$ is required to interpret them as part of amplitudes related to some physical processes. A brief study of the limits shows that the $Z$ functions are continuous at these points and the $Y$ functions are discontinuous at the considered points.

\section{A.1. Functions $Z_{k}\left(\lambda^{2}, p^{2}\right)$ and $Y_{k}\left(\lambda^{2}, p^{2}\right)$}

The results in this section are useful for calculating processes involving fermion loops or theories with a single mass. For this purpose we replace $\lambda_{1}^{2}=\lambda_{2}^{2}=\lambda^{2}$ in the functions $Y$ and $Z$. As we have equal masses there are two different situations, which we will separate into region $a$ and $b$. Region $a$ when $p^{2}<4 \lambda^{2}$ and region $b$ when $p^{2}>4 \lambda^{2}$. With that we can finally write the functions $Z$ s and $Y$ s as:

$$
\begin{aligned}
Z_{0}^{a}\left(\lambda^{2}, p^{2}\right)= & \frac{\sqrt{4 \lambda^{2}-p^{2}}}{|p|} \\
& \times\left[2 \arctan \left(\frac{\sqrt{4 \lambda^{2}-p^{2}}}{|p|}\right)+\pi\right]-2 \\
Z_{0}^{b}\left(\lambda^{2}, p^{2}\right)= & -\frac{\sqrt{p^{2}-4 \lambda^{2}}}{|p|} \\
& \times\left[\ln \left(\frac{|p|-\sqrt{p^{2}-4 \lambda^{2}}}{|p|+\sqrt{p^{2}-4 \lambda^{2}}}\right)+i \pi\right]-2
\end{aligned}
$$

The other functions can be written in terms of $Z_{0}$ as we can verify, namely:

$$
\begin{aligned}
Z_{0} & =-\frac{F \cdot G}{2 p^{2}}-2 \\
Z_{1} & =-\frac{1}{2}\left(\frac{F \cdot G}{2 p^{2}}+2\right)=\frac{Z_{0}}{2} \\
Z_{2} & =-\frac{1}{3} \frac{F \cdot G}{2 p^{2}}\left(1-\frac{\lambda^{2}}{p^{2}}\right)+\frac{2 \lambda^{2}}{3 p^{2}}-\frac{13}{18} \\
& =Z_{0} / 3\left(1-\frac{\lambda^{2}}{p^{2}}\right)-\frac{1}{18}
\end{aligned}
$$

In the same way for the $Y$ functions we have:

$$
\begin{aligned}
& Y_{0}=\frac{\lambda^{2}}{2} \frac{G}{F} \\
& Y_{1}=\frac{\lambda^{2}}{p^{2}}\left(2 Y_{0}-1\right) \\
& Y_{2}=\left(2-3 \frac{\lambda^{2}}{p^{2}}-2 \frac{\lambda^{2}}{p^{4}}\right) Y_{0}-\frac{\lambda^{2}}{2 p^{2}}
\end{aligned}
$$

where

$$
\begin{aligned}
& Y_{0}^{a}\left(\lambda^{2}, p^{2}\right)=\frac{\lambda^{2}}{2}\left\{\frac{2 \pi+4 \arctan \left(\frac{\sqrt{4 \lambda^{2}-p^{2}}}{|p|}\right)}{|p| \sqrt{4 \lambda^{2}-p^{2}}}\right\} \\
& Y_{0}^{b}\left(\lambda^{2}, p^{2}\right)=\frac{\lambda^{2}}{2}\left\{\frac{2 \ln \left(\frac{|p|-\sqrt{p^{2}-4 \lambda^{2}}}{\left.|p|+\sqrt{p^{2}-4 \lambda^{2}}\right)+2 i \pi}\right.}{|p| \sqrt{p^{2}-4 \lambda^{2}}}\right\}
\end{aligned}
$$




\section{A.2. Functions $Z_{k}\left(p^{2} \ll 1\right)$ and $Y_{k}\left(p^{2} \ll 1\right)$}

In this section we will study the behavior of the functions $Z s$ and $Y s$, equations (4) to (9), at the limit where the four-vector moment-energy module tends to zero and see that these functions are well behaved at the origin.

If we expand function $Z_{0}$ and function $Y_{1}$ to small values of $p^{2}$, as an example, and then take the limit, we see that these functions are well behaved at the origin. We have the expression below, considering we only have up to $p^{4}$ :

$$
\begin{aligned}
Z_{0}\left(\lambda_{1}^{2}, \lambda_{2}^{2}, p^{2} \ll 1\right) & \\
= & -\left[\frac{\lambda_{1}^{2} \lambda_{2}^{2}}{\left(\lambda_{1}^{2}-\lambda_{2}^{2}\right)^{3}} p^{2}+\frac{\left(\lambda_{1}^{2}+\lambda_{2}^{2}\right)}{2\left(\lambda_{1}^{2}-\lambda_{2}^{2}\right)}+\frac{1}{2}\right] \ln \left(\frac{\lambda_{2}^{2}}{\lambda_{1}^{2}}\right) \\
& -\frac{\left(\lambda_{1}^{2}+\lambda_{2}^{2}\right)^{2}+4 \lambda_{1}^{2} \lambda_{2}^{2}}{2\left(\lambda_{1}^{2}-\lambda_{2}^{2}\right)^{4}} p^{4}-\frac{\left(\lambda_{1}^{2}+\lambda_{2}^{2}\right)}{2\left(\lambda_{1}^{2}-\lambda_{2}^{2}\right)^{2}} p^{2}-1
\end{aligned}
$$

$$
\begin{aligned}
& Y_{1}\left(\lambda_{1}^{2}, \lambda_{2}^{2}, p^{2} \ll 1\right) \\
& =\lambda_{2}^{2}\left\{\frac{p^{2}\left[\left(\lambda_{1}^{2}+\lambda_{2}^{2}\right)^{2}+4 \lambda_{1}^{2} \lambda_{2}^{2}\right]-\left(\lambda_{1}^{2}+\lambda_{2}^{2}\right)\left(\lambda_{1}^{2}-\lambda_{2}^{2}\right)^{2}}{2\left[2 p^{4} \lambda_{1}^{2} \lambda_{2}^{2}+p^{2}\left(\lambda_{1}^{2}+\lambda_{2}^{2}\right)\left(\lambda_{1}^{2}-\lambda_{2}^{2}\right)^{2}-\left(\lambda_{1}^{2}-\lambda_{2}^{2}\right)^{4}\right]}\right. \\
& \quad-\frac{\lambda_{1}^{2} \lambda_{2}^{2}\left(\lambda_{1}^{2}-\lambda_{2}^{2}\right)}{\left[2 p^{4} \lambda_{1}^{2} \lambda_{2}^{2}+p^{2}\left(\lambda_{1}^{2}+\lambda_{2}^{2}\right)\left(\lambda_{1}^{2}-\lambda_{2}^{2}\right)^{2}-\left(\lambda_{1}^{2}-\lambda_{2}^{2}\right)^{4}\right]} \\
& \left.\quad \times \ln \left(\frac{\lambda_{2}^{2}}{\lambda_{1}^{2}}\right)\right\}
\end{aligned}
$$

Taking the limit $p^{2}=0$ we have:

$$
\begin{aligned}
& Z_{0}\left(\lambda_{1}^{2}, \lambda_{2}^{2}, 0\right)=-\left(\frac{\left(\lambda_{1}^{2}+\lambda_{2}^{2}\right)}{2\left(\lambda_{1}^{2}-\lambda_{2}^{2}\right)}+\frac{1}{2}\right) \ln \left(\frac{\lambda_{2}^{2}}{\lambda_{1}^{2}}\right)-1 \\
& Y_{1}\left(\lambda_{1}^{2}, \lambda_{2}^{2}, 0\right)=\frac{\lambda_{2}^{2}\left(\lambda_{1}^{2}+\lambda_{2}^{2}\right)}{2\left(\lambda_{1}^{2}-\lambda_{2}^{2}\right)^{2}}+\frac{\lambda_{1}^{2} \lambda_{2}^{4}}{\left(\lambda_{1}^{2}-\lambda_{2}^{2}\right)^{3}} \ln \left(\frac{\lambda_{2}^{2}}{\lambda_{1}^{2}}\right)
\end{aligned}
$$

We can see by these expressions that the coefficient of $p^{2}$ in $Z_{0}$ expansion is just $Y_{1}\left(\lambda_{1}^{2}, \lambda_{2}^{2}, 0\right)$ which is in accordance with the equation A.3. The other functions $Z_{k}$ and $Y_{k}$ can be obtained by replacing $p^{2}=0$ and directly integrating A.1 and A.2.

\section{A.3. Functions $Z_{k}\left(\lambda_{1}^{2} \gg 1\right)$ and $Y_{k}\left(\lambda_{1}^{2} \gg 1\right)$}

Finally, we show the behavior of these functions at the limit $\lambda \rightarrow \infty$. These results are useful mainly to calculate divergent amplitudes using the regularization of PauliVillars, in which we introduce a regularization parameter $\lambda$ playing the role of one of the masses. Taking this limit for the functions $Z_{0}$ and $Y_{0}$ we have:

$$
\lim _{\lambda_{1}^{2} \rightarrow \infty} Z_{0}\left(\lambda_{1}^{2}, \lambda_{2}^{2}, p^{2}\right)=\lim _{\lambda_{1}^{2} \rightarrow \infty}\left(1-\frac{\lambda_{2}^{2}}{2 p^{2}}\right) \ln \left(\frac{\lambda_{2}^{2}}{\lambda_{1}^{2}}\right)-2
$$

and

$$
\lim _{\lambda_{1}^{2} \rightarrow \infty} Y_{0}\left(\lambda_{1}^{2}, \lambda_{2}^{2}, p^{2}\right)=\lim _{\lambda_{1}^{2} \rightarrow \infty}-\frac{\lambda_{2}^{2}}{2 p^{2}} \ln \left(\frac{\lambda_{2}^{2}}{\lambda_{1}^{2}}\right)
$$

that diverge logarithmically. The other functions $Z_{k}$ and $Y_{k}$ will have the same behavior as can be easily verified.

\section{References}

[1] N.N. Bogoliubov and O. Parasiuk, Acta Math. 97, 227 (1957).

[2] Hepp, K., Comm. Math. Phys. 2, 301 (1966).

[3] Zimmerman, W. Comm. Math. Phys. 15, 208 (1969).

[4] R.P. Feynman, Quantum Electrodynamics (AddisonWesley Publishing Company, New York, 1961).

[5] R.P. Feynman, Phys. Rev. 76, 769 (1949).

[6] G.'t Hooft and M. Veltman, Nucl. Phys. B44, 189 (1972).

[7] G.'t Hooft and M. Veltman, Nucl. Phys. B50 318 (1972).

[8] P.F. Villars, Rev. Mod. Phys. 21, 434 (1949).

[9] M. Sampaio, A. Scarpelli, B Hiller, A. Brizola, M.C. Nemes and S.R. Gobira, Physical Review D65, 125023 (2002).

[10] S.R. Gobira and M.C. Nemes, International Journal of Theoretical Physics 42, 11 (2003).

[11] S.R. Gobira, O.A. Battistel and M.C. Nemes, Braz. Journ. Phys. 30, 610 (2000).

[12] S.R. Gobira and M. C. Nemes, Braz. Journ. Phys. 35 (2005) 652.

[13] S. Weinberg, The Quantum Theory of Fields (Cambridge University Press, Cambridge, 1995).

[14] W.H. Furry, Phys. Rev. 51, 125 (1937). 